1 Hacettepe Journal of Mathematics and Statistics

$\bigcap$ Volume 45 (5) (2016), $1321-1328$

\title{
On commutativity of prime gamma rings with derivation
}

\author{
Okan Arslan*† and Hatice Kandamar ${ }^{\ddagger}$
}

\begin{abstract}
Let $M$ be a weak Nobusawa $\Gamma$-ring and $\gamma$ be a nonzero element of $\Gamma$. In this paper, we find a relation between $\Gamma$-rings and rings, and give some commutativity conditions on $\Gamma$-rings by using this relation. Also, we prove that any $\Gamma$-ring $M$ in the sense of Nobusawa with a nonzero element $\gamma$ in the center of $M$-ring $\Gamma$ is $\gamma$-prime if and only if $M$ is $\Gamma$-prime. As a consequence, we show that the semiprimeness (semisimpleness) of the ring $(M,+, \cdot \gamma)$ for any $\gamma \in \Gamma$ implies the $\Gamma$ semiprimeness $(\Gamma$-semisimpleness $)$ of the $\Gamma$-ring $M$.
\end{abstract}

Keywords: gamma ring, prime $\Gamma$-ring, $k$-derivation, commutativity, $\gamma$-radical. 2000 AMS Classification: AMS Primary 16N60; Secondary 16W25, 16 Y99

Received : 10.03.2015 Accepted: 21.10.2015 Doi : 10.15672/HJMS.20164514286

\section{Introduction}

Let $M$ and $\Gamma$ be additive Abelian groups. $M$ is said to be a $\Gamma$-ring in the sense of Barnes [3] if there exists a mapping $M \times \Gamma \times M \rightarrow M$ satisfying these two conditions for all $a, b, c \in M, \alpha, \beta \in \Gamma$ :

(1) $(a+b) \alpha c=a \alpha c+b \alpha c$ $a(\alpha+\beta) c=a \alpha c+a \beta c$ $a \alpha(b+c)=a \alpha b+a \alpha c$

(2) $(a \alpha b) \beta c=a \alpha(b \beta c)$

In addition, if there exists a mapping $\Gamma \times M \times \Gamma \rightarrow \Gamma$ such that the following axioms hold for all $a, b, c \in M, \alpha, \beta \in \Gamma$ :

(3) $(a \alpha b) \beta c=a(\alpha b \beta) c$

*Department of Mathematics, Adnan Menderes University, Aydın, Turkey, Email: oarslann@icloud.com

$\dagger$ Corresponding Author.

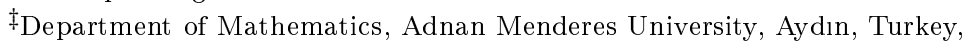
Email: hkandamar@adu.edu.tr 
(4) $a \alpha b=0$ for all $a, b \in M$ implies $\alpha=0$, where $\alpha \in \Gamma$

then $M$ is called a $\Gamma$-ring in the sense of Nobusawa [17]. If a $\Gamma$-ring $M$ in the sense of Barnes satisfies only the condition (3), then it is called weak Nobusawa $\Gamma$-ring [11].

We assume that all gamma rings in this paper are weak Nobusawa gamma ring unless otherwise specified.

Let $M$ be a $\Gamma$-ring. $M$ is said to be a $\Gamma$-prime gamma ring if $a \Gamma M \Gamma b=0$ with $a, b \in M$ implies either $a=0$ or $b=0$ [15]. $M$ is $\Gamma$-simple if $M \Gamma M \neq 0$ and $M$ has no ideals (0) and $M$ itself [15].

$C_{M}=\{\alpha \in \Gamma \mid \alpha m \beta=\beta m \alpha, \forall m \in M, \beta \in \Gamma\}$ is called the center of $M$-ring $\Gamma$ and $C_{\gamma}=\{c \in M \mid c \gamma m=m \gamma c, \forall m \in M\}$ with $\gamma \in \Gamma$ is called the $\gamma$-center of $\Gamma$-ring $M$.

Recall that from [9], an additive mapping $d: M \rightarrow M$ is called a derivation on $M$ if $d(a \alpha b)=d(a) \alpha b+a \alpha d(b)$ for all $a, b \in M$ and $\alpha \in \Gamma$. Note that $d=0$ when $d$ is defined on a prime weak Nobusawa $\Gamma$-ring $M$. So, in this paper we consider $k$-derivations that has been defined by Kandamar [10] on any gamma ring $M$.

In this work, we first obtain some commutativity conditions on the $\gamma$-prime $\Gamma$-ring $M$ with $k$-derivations and prove that $M$ is $\gamma$-prime if and only if $M$ is $\Gamma$-prime where $\gamma$ is a nonzero element in the center of $M$-ring $\Gamma$ in the sense of Nobusawa. Then, we also show that if there exists a nonzero element $\gamma$ in $C_{M}$ in a Nobusawa $\Gamma$-ring $M$, then $(0)$ is $\Gamma$-prime ideal if and only if $(0)$ is $\gamma$-prime ideal. Finally, we study the relation between semiprimeness (semisimpleness) of the ring $\left(M,+,{ }^{\prime} \gamma\right)$ and $\Gamma$-semiprimeness $(\Gamma$ semisimpleness) of the $\Gamma$-ring $M$ where $\gamma \in \Gamma$.

\section{Relation between $\Gamma$-rings and rings up to $\gamma$}

We now give some definitions that have been firstly defined by Arslan and Kandamar in [1].

2.1. Definition. Let $M$ be a $\Gamma$-ring, $\gamma$ be a nonzero element of $\Gamma$ and $I$ be an additive subgroup of $M$.

(i) $M$ is said to be $\gamma$-commutative if $x \gamma y=y \gamma x$ for all $x, y \in M$.

(ii) $I$ is said to be a $\gamma$-subring of $M$ if $x \gamma y \in I$ for all $x, y \in I$.

(iii) $I$ is said to be a $\gamma$-left ideal(resp. $\gamma$-right ideal) of $M$ if $m \gamma a \in I$ (resp. $a \gamma m \in I$ ) for all $m \in M, a \in I$. If $I$ is both $\gamma$-left and $\gamma$-right ideal then $I$ is called a $\gamma$-ideal of $M$.

(iv) $I$ is said to be a $\gamma$-prime ideal if $A \gamma B$ implies $A \subseteq I$ or $B \subseteq I$ for any $\gamma$-ideals $A$ and $B$ of $M$.

(v) $I$ is said to be a $\gamma$-Lie ideal of $M$ if $[x, m]_{\gamma}=x \gamma m-m \gamma x \in I$ for all $x \in I$ and $m \in M$.

2.2. Definition. A $\Gamma$-ring $M$ is called a $\gamma$-prime gamma ring if there exists a nonzero element $\gamma$ in $\Gamma$ such that $a \gamma M \gamma b=0$ with $a, b \in M$ implies either $a=0$ or $b=0$.

2.3. Definition. A $\Gamma$-ring $M$ is called a $\gamma$-simple if $M \gamma M \neq 0$ and $M$ has no $\gamma$-ideal besides the (0) and itself.

2.4. Lemma. Let $M$ be a $\Gamma$-ring. Then the following holds:

(i) If $M$ is a $\gamma$-prime gamma ring, then $M$ is $\Gamma$-prime.

(ii) If $M$ is a $\gamma$-simple gamma ring, then $M$ is $\Gamma$-simple.

Proof. (i) Let $M$ be a $\gamma$-prime gamma ring and $a \Gamma M \Gamma b=0$ for any $a, b \in M$. Therefore, we have $a \gamma M \gamma b=0$. Since $M$ is a $\gamma$-prime gamma ring, we get $a=0$ or $b=0$. Hence, the $\gamma$-primeness of $\mathrm{M}$ implies the $\Gamma$-primeness of $M$. 
(ii) It is clear from the definitions of $\gamma$-simple and $\Gamma$-simple gamma rings.

2.5. Proposition. Let $M$ be $a \Gamma$-ring and $\gamma$ be a nonzero element of $\Gamma$. Then the Abelian group $M$ with a binary operation $\cdot_{\gamma}$ defined by $a \cdot{ }_{\gamma} b=a \gamma b$ for all $a, b \in M$ is a ring.

Proof. It is clear from the definition of the gamma ring.

According to the Proposition 2.5, the Abelian group $M$ can be made into a ring by defining binary operations for all $\gamma \in \Gamma$. We denote this ring by $(M,+, \cdot \gamma)$.

It is obvious that a $\gamma$-ideal of a $\Gamma$-ring $M$ is an ideal of the ring $(M,+, \cdot \gamma)$. Conversely, every ideal of the ring $(M,+, \cdot \gamma)$ is a $\gamma$-ideal of the $\Gamma$-ring $M$. Similarly $\gamma$-Lie ideals of the $\Gamma$-ring $M$ and Lie ideals of the ring $(M,+, \cdot \gamma)$ is same. Also, if $d$ is a $k$-derivation of the $\Gamma$-ring $M$ and $k(\gamma)=0$, then $d$ is a derivation of the ring $(M,+, \cdot \gamma)$. Thus, we can adapt all of the known results for the ring $(M,+, \cdot \gamma)$ to the $\Gamma$-ring $M$. For instance, the commutativity of the ring $\left(M,+,{ }^{\prime} \gamma\right)$ is equal to the $\gamma$-commutativity of the $\Gamma$-ring $M$. Similarly one can say the primeness (semiprimeness) of the ring $(M,+, \cdot \gamma)$ is the same as the $\gamma$-primeness $(\gamma$-semiprimeness) of the $\Gamma$-ring $M$. We give some results below.

2.6. Theorem. Let $M$ be a $\gamma$-prime gamma ring and $d_{1}, d_{2}$ be nonzero $k_{1}, k_{2}$-derivations of $M$ such that $k_{1}(\gamma)=k_{2}(\gamma)=0$ respectively. If char $M \neq 2$ and $d_{1} d_{2}$ is $k_{1} k_{2}$-derivation of $M$, then $d_{1}=0$ or $d_{2}=0$.

Proof. By the hypothesis $d_{1} \neq 0, d_{2} \neq 0$ and $d_{1} d_{2}$ are derivations of the prime ring $\left(M,+, \cdot_{\gamma}\right)$. Also the characteristic of the ring $\left(M,+, \cdot_{\gamma}\right)$ is different from 2 . Therefore by $\left[18\right.$, Theorem 1] one of the derivations $d_{1}$ and $d_{2}$ is zero in the ring $\left(M,+, \cdot_{\gamma}\right)$.

2.7. Corollary. Let $M$ be a $\gamma$-prime gamma ring of characteristic not 2 and $d$ be a 0 -derivation of $M$ such that $d^{2}=0$. Then $d=0$.

Proof. Let $M$ is a $\gamma$-prime gamma ring. Then $M$ is a $\Gamma$-prime gamma ring by Lemma 2.4. Since $d^{2}=0$ is a derivation on $M$, we get $d=0$ by Theorem 2.6.

2.8. Theorem. Let $M$ be a gamma ring and $d$ be a $k$-derivation of $M$ such that $k(\gamma)=0$ and $d^{3} \neq 0$. Then the $\gamma$-subring generated by $d(m)$ for all $m$ in $M$ contains a nonzero $\gamma$-ideal of $M$.

Proof. Since $d$ is a derivation of the $\operatorname{ring}(M,+, \cdot \gamma)$ and $d^{3} \neq 0$, the subring generated by $d(m)$ for all $m$ in $M$ contains a nonzero ideal of $\left(M,+,{ }^{\cdot} \gamma\right)$ by [6, Theorem 1]. Therefore the $\gamma$-subring generated by $d(m)$ for all $m$ in $M$ contains a nonzero $\gamma$-ideal of $M$.

2.9. Corollary. Let $M$ be a $\Gamma$-ring, $d$ be a nonzero 0 -derivation on $M$ such that $d^{3} \neq 0$. Then, the subring $A$ of $M$ generated by all $d(a \alpha b)$, with $\alpha \in \Gamma$ and $a, b \in M$, contains $a$ nonzero ideal of $M$.

Another proof of Corollary 2.9 can be found in [19].

2.10. Theorem. Let $M$ be a $\gamma$-prime gamma ring and $d$ be a nonzero $k$-derivation of $M$ such that $k(\gamma)=0$. Then $M$ is $\gamma$-commutative if one of the following conditions holds:

(i) $[a, d(a)]_{\gamma} \in C_{\gamma}$ for all $a \in M$.

(ii) $\operatorname{char} M \neq 2$ and $[d(M), d(M)]_{\gamma} \subset C_{\gamma}$.

(iii) $\operatorname{char} M \neq 2$ and $d^{2}(M) \subset C_{\gamma}$.

(iv) $d_{1}, d_{2}$ are nonzero $k_{1}, k_{2}$-derivations of $M$ such that $k_{1}(\gamma)=k_{2}(\gamma)=0$ respectively, $\operatorname{char} M \neq 2$ and $d_{1} d_{2}(M) \subset C_{\gamma}$. 
Proof. (i) By the hypothesis $d$ is a nonzero derivation of the prime $\operatorname{ring}\left(M,+,{ }_{\gamma}\right)$. Since $[a, d(a)]$ is in the center of the $\operatorname{ring}\left(M,+,{ }_{\gamma}\right)$ for all $a \in M$, the ring $\left(M,+,{ }_{\gamma}\right)$ is commutative by [18, Theorem 2]. Therefore the gamma ring $M$ is $\gamma$-commutative since commutativity of $(M,+, \cdot \gamma)$ requires $\gamma$-commutativity of $\Gamma$-ring $M$.

(ii) By the hypothesis $d$ is a nonzero derivation of the prime ring $\left(M,+, \cdot_{\gamma}\right)$, the characteristic of the ring $M$ is different from 2 and $[d(M), d(M)]_{\gamma}$ is contained in the center of the ring $M$. Hence $M$ is commutative as a ring by [13, Theorem 2]. Therefore $M$ is $\gamma$-commutative.

(iii) By the hypothesis $d$ is a nonzero derivation of the prime ring $(M,+, \cdot \gamma)$, the characteristic of the ring $M$ is different from 2 and $d^{2}(M)$ is contained in the center of the $\operatorname{ring} M$. Hence $M$ is commutative as a ring by [13, Theorem 3]. Therefore $M$ is $\gamma$-commutative.

(iv) By the hypothesis $d_{1}$ and $d_{2}$ are nonzero derivations of the prime $\operatorname{ring}\left(M,+,{ }^{\cdot} \gamma\right)$. Also the characteristic of the ring $\left(M,+,{ }_{\gamma}\right)$ is different from 2 and $d_{1} d_{2}(M)$ is contained in the center of the ring $M$. Hence $M$ is commutative as a ring by [13, Theorem 4]. Therefore $M$ is $\gamma$-commutative.

2.11. Corollary. Let $M$ be a $\gamma$-prime gamma ring for all nonzero elements $\gamma$ in $\Gamma$ and $d$ be a nonzero 0-derivation on $M$. Then $M$ is $\Gamma$-commutative if one of the following conditions holds:

(i) $[a, d(a)]_{\gamma} \in C_{\gamma}$ for all $a \in M$ and $\gamma \in \Gamma$.

(ii) $\operatorname{char} M \neq 2$ and $[d(M), d(M)]_{\gamma} \subset C_{\gamma}$ for all $\gamma \in \Gamma$.

(iii) $\operatorname{char} M \neq 2$ and $d^{2}(M) \subset C_{\gamma}$ for all $\gamma \in \Gamma$.

(iv) $d_{1}, d_{2}$ are nonzero 0-derivations of $M$, char $M \neq 2$ and $d_{1} d_{2}(M) \subset C_{\gamma}$ for all $\gamma \in \Gamma$.

2.12. Theorem. Let $M$ be a $\gamma$-prime gamma ring of characteristic not 2 and $U$ be a $\gamma$-Lie ideal of $M$. If $U \nsubseteq C_{\gamma}$, then there exists a $\gamma$-ideal $K$ of $M$ such that $[K, M]_{\gamma} \subseteq U$ but $[K, M]_{\gamma} \nsubseteq C_{\gamma}$.

Proof. $U$ is a Lie ideal of the prime ring $\left(M,+,{ }^{\prime} \gamma\right)$ that is not contained in the center of the ring $M$ and the characteristic of the ring $M$ is different from 2 by hypothesis. Hence, there exists an ideal $K$ of $(M,+, \cdot \gamma)$ such that $[K, M] \subseteq U$ and $[K, M]$ is not contained in the center of the $(M,+, \cdot \gamma)$ by [4, Lemma 1]. Therefore, there exists an ideal $K$ of $\Gamma$-ring $M$ such that $[K, M]_{\gamma} \subseteq U$ but $[K, M]_{\gamma} \nsubseteq C_{\gamma}$.

2.13. Theorem. Let $M$ be a $\gamma$-prime gamma ring of characteristic not 2 and $U$ be $a$ $\gamma$-Lie ideal of $M$ such that $U \nsubseteq C_{\gamma}$. If $d_{1}, d_{2}$ are nonzero $k_{1}, k_{2}$-derivations of $M$ such that $k_{1}(\gamma)=k_{2}(\gamma)=0$ respectively and $d_{1} d_{2}(U)=0$, then $d_{1}=0$ or $d_{2}=0$.

Proof. By the hypothesis, $d_{1}$ and $d_{2}$ are nonzero derivations of the prime $\operatorname{ring}\left(M,+,{ }^{\cdot} \gamma\right)$ and $U$ is a Lie ideal of $M$ that is not contained in the center of the ring $M$. Also the characteristic of the $\operatorname{ring}\left(M,+,{ }_{\gamma}\right)$ is different from 2 and $d_{1} d_{2}(U)=0$. Hence $d_{1}=0$ or $d_{2}=0$ by [4, Theorem 4$]$.

2.14. Theorem. Let $M$ be a $\gamma$-prime gamma ring of characteristic not $2, U$ be a $\gamma$-Lie ideal of $M$ and $d$ be a $k$-derivation of $M$ such that $k(\gamma)=0$. Then $U$ is contained in the $\gamma$-center of $M$ if one of the following conditions holds:

(i) $d^{2}(U)=0$.

(ii) $d \neq 0$ and $d^{2}(U) \subset C_{\gamma}$. 
(iii) $d_{1}, d_{2}$ are nonzero $k_{1}, k_{2}$-derivations of $M$ such that $k_{1}(\gamma)=k_{2}(\gamma)=0$ respectively and $d_{1} d_{2}(U) \subset C_{\gamma}$.

Proof. It is similar to the proof of Theorem 2.10 .

2.15. Corollary. Let $M$ be a $\gamma$-prime gamma ring of characteristic not 2 for all nonzero elements $\gamma$ in $\Gamma, U$ be a $\gamma$-Lie ideal of $M$ and $d$ be a 0 -derivation of $M$. Then $U$ is contained in the center of $M$ if one of the following conditions holds:

(i) $d^{2}(U)=0$.

(ii) $d \neq 0$ and $d^{2}(U) \subset C_{\gamma}$ for all $\gamma \in \Gamma$.

(iii) $d_{1}, d_{2}$ are nonzero 0 -derivations of $M$ and $d_{1} d_{2}(U) \subset C_{\gamma}$ for all $\gamma \in \Gamma$.

2.16. Theorem. Let $M$ be a $\gamma$-prime gamma ring of characteristic not 2 and $U$ be a $\gamma$-Lie ideal of $M$ such that $U \nsubseteq C_{\gamma}$. If $d_{1}$ and $d_{2}$ are nonzero $k_{1}$ and $k_{2}$-derivations of $M$ such that $k_{1}(\gamma)=k_{2}(\gamma)=0$ respectively and $d_{1} d_{2}(U) \subset C_{\gamma}$, then $d_{1}=0$ or $d_{2}=0$.

Proof. By the hypothesis $d_{1}$ and $d_{2}$ are nonzero derivations of the prime $\operatorname{ring}(M,+, \cdot \gamma)$ and $U$ is a Lie ideal of $M$ that is not contained in the center of $M$. Also the characteristic of the ring $\left(M,+,{ }^{\prime} \gamma\right)$ is different from 2 and $d_{1} d_{2}(U)$ is contained in the center of $M$. Hence $d_{1}=0$ or $d_{2}=0$ by [2, Theorem 6$]$.

\section{3. $\gamma$-Radicals of Gamma Rings}

Radicals of $\Gamma$-rings has been investigated by a number of authors. Barnes [3] defined prime radicals and proved some properties for gamma rings by methods similar to those of McCoy[16]. Coppage and Luh [5] introduced the notions of Jacobson radical, Levitzki nil radical, nil radical and strongly nilpotent radical for $\Gamma$-rings and Barnes' prime radical was studied further. Kyuno [12] also studied prime radicals of gamma rings and showed relations between radicals of gamma rings and radicals of its operator rings.

We define $\gamma$-prime radical, strongly $\gamma$-nilpotent radical, $\gamma$-Levitzki nil radical and $\gamma$ Jacobson radical for $\Gamma$-rings and show their relations with the radicals of $\Gamma$-rings in the literature.

Let $M$ be a gamma ring and $S \subseteq M . S$ is said to be a $\gamma-m$-system if $S=\emptyset$ or $(a)_{\gamma} \gamma(b)_{\gamma} \cap S \neq \emptyset$ for any $a, b \in M$. Here, $(a)_{\gamma}$ is the set of all elements of the form $k a+m \gamma a+a \gamma x+\sum_{i=1}^{n} u_{i} \gamma a \gamma v_{i}$ for $k \in \mathbb{Z}, n \in \mathbb{N}, m, x, u_{i}, v_{i} \in M$.

Proofs of the below results are obvious from the relation given in Section 2. So we omit their proofs.

3.1. Proposition. Let $M$ be a gamma ring and $P$ be $a$-ideal of $M$. Then $P$ is $a$ $\gamma$-prime ideal if and only if the complement of $P$ is a $\gamma$-m-system.

Let $A$ be a $\gamma$-ideal of a $\Gamma$-ring $M$. Then the set of all elements $m$ in $M$ such that every $\gamma$ - $m$-system in $M$ which contains $m$ meets $A$ is called $\gamma$-prime radical of the $\gamma$-ideal $A$ and is denoted by $\mathfrak{B}_{\gamma}(A)$. $\gamma$-prime radical of zero $\gamma$-ideal is called $\gamma$-prime radical of the $\Gamma$-ring $M$ and is denoted by $\mathfrak{B}_{\gamma}(M)$. In fact, the prime radical of the $\operatorname{ring}\left(M,+,{ }_{\gamma}\right)$ is equal to $\mathfrak{B}_{\gamma}(M)$.

3.2. Theorem. If $A$ is a $\gamma$-ideal in the $\Gamma$-ring $M$, then $\mathfrak{B}_{\gamma}(A)$ coincides with the intersection of all the $\gamma$-prime ideals in $M$ which contain $A$.

3.3. Corollary. $\gamma$-prime radical of a $\Gamma$-ring $M$ is the intersection of all the $\gamma$-prime ideals in $M$. 
An element $a$ in $M$ is called strongly $\gamma$-nilpotent if there exists a positive integer $n$ such that $(a \gamma)^{n} a=0$. A subset $L$ of $M$ is called strongly $\gamma$-nil if all of the elements in $L$ are strongly $\gamma$-nilpotent. A subset $S$ of $M$ is called strongly $\gamma$-nilpotent if there exists a positive integer $m$ such that $(S \gamma)^{m} S=0$.

The strongly $\gamma$-nilpotent radical of $M$ is the sum of all strongly $\gamma$-nilpotent ideals of $M$ and is denoted by $\mathfrak{S}_{\gamma}(M)$.

3.4. Proposition. If $A$ and $B$ are any strongly $\gamma$-nilpotent ideals in a $\Gamma$-ring $M$, then $A+B$ is also a strongly $\gamma$-nilpotent ideal in $M$.

3.5. Corollary. The strongly $\gamma$-nilpotent radical of a $\Gamma$-ring $M$ is a strongly $\gamma$-nil ideal in $M$.

A subset $S$ of $M$ is called $\gamma$-locally nilpotent if for any finite subset $F$ of $S$ there exists a positive integer $n$ such that $(F \gamma)^{n} F=0$.

The $\gamma$-Levitzki nil radical of $M$ is the sum of all $\gamma$-locally nilpotent ideals of $M$ and is denoted by $\mathfrak{L}_{\gamma}(M)$.

An element $a$ in $M$ is called $\gamma$-right quasi regular if there exist $b \in M$ such that $a+b+a \gamma b=0$. A subset $S$ of $M$ is called $\gamma$-right quasi regular if all of the elements in $S$ are $\gamma$-right quasi regular.

The $\gamma$-Jacobson radical of $M$ is the set of all $a \in M$ such that the principal $\gamma$-ideal generated by $a$ is $\gamma$-right quasi regular and is denoted by $\mathfrak{J}_{\gamma}(M)$. In fact, the Jacobson radical of the ring $\left(M,+,{ }_{\gamma}\right)$ is equal to $\mathfrak{J}_{\gamma}(M)$.

\section{Main Results}

Not all of the properties of a ring holds for a gamma ring. For example, let $d$ be a $k$-derivation of $\gamma$-prime gamma ring $M$ of characteristic not 2 . If $k(\gamma) \neq 0$, then the hypothesis $d^{2}=0$ does not imply $d=0$.

4.1. Example. Let $M=\left\{\left(\begin{array}{ccc}a & b & a \\ c & r & c\end{array}\right) \mid a, b, c, r \in \mathbb{Z}\right\}, \Gamma$ be the set of all $3 \times 2$ matrices over $\mathbb{Z}$ and $\gamma=\left(\begin{array}{cc}0 & 0 \\ 0 & -1 \\ 1 & 0\end{array}\right) \in \Gamma$. Then, $M$ is a $\gamma$-prime $\Gamma$-ring of characteristic not 2. Define $d: M \rightarrow M, d\left(\begin{array}{ccc}a & b & a \\ c & r & c\end{array}\right)=\left(\begin{array}{ccc}-b & 0 & -b \\ -r & 0 & -r\end{array}\right)$ and $k: \Gamma \rightarrow \Gamma$, $k\left(\begin{array}{ll}u_{11} & u_{12} \\ u_{21} & u_{22} \\ u_{31} & u_{32}\end{array}\right)=\left(\begin{array}{cc}0 & 0 \\ u_{11}+u_{31} & u_{12}+u_{32} \\ 0 & 0\end{array}\right)$

It can be shown that $d$ is a $k$-derivation and $k(\gamma) \neq 0$. Moreover, it is easy to see that $d \neq 0$ but $d^{2}=0$.

This example also shows that if $d$ is a $k$-derivation on the $\Gamma$-prime gamma ring of characteristic not 2 such that $d^{2}=0$, then $d$ may not be the zero derivation. In such a case, $k^{2}$ must be equal to zero as proved in the next theorem.

4.2. Theorem. Let $M$ be a $\gamma$-prime gamma ring in the sense of Nobusawa of characteristic not 2 and $d$ be a $k$-derivation. If $d^{2}=0$, then either $d=0$ or $k^{2}=0$.

Proof. Let $k(\gamma)=0$. Then, the $k$-derivation $d$ on $M$ is also a derivation for the ring $(M,+, \cdot \gamma)$. Therefore, $d=0$ by [18, Theorem 1]. Now, let $k(\gamma) \neq 0$. By hypothesis we have $d^{2}(d(x) \beta d(y))=0$ for all $x, y \in M$ and $\beta \in \Gamma$. Expanding this we get $d(x) k^{2}(\beta) d(y)=0$. Replacing $\beta$ by $\beta d(z) \alpha$ we have $d(x) k(\beta) d(z) k(\alpha) d(y)=0$ since $\operatorname{char} M \neq 2$. Replacing $\beta$ by $\beta d(m) \delta$ we get $d(x) k(\beta) d(m)=0$ since $M$ is $\Gamma$-prime 
Nobusawa $\Gamma$-ring by Lemma 2.4. If we replace $x$ by $d(x) \alpha y$ in the last equation we have $d(x) k(\alpha) y=0$ or $z k(\beta) d(m)=0$. If $d(x) k(\alpha) y=0$, then replacing $\alpha$ by $\alpha m k(\delta)$ we get $d(x) \alpha m k^{2}(\delta) y=0$ for all $x, m, y \in M$ and $\alpha, \delta \in \Gamma$. Then, $d=0$ or $k^{2}=0$ since $M$ is a prime Nobusawa $\Gamma$-ring. If we consider the case $z k(\beta) d(m)=0$, same result can be obtained similarly.

4.3. Theorem. Let $M$ be a $\Gamma$-ring in the sense of Nobusawa and $\gamma$ be a nonzero element of $\Gamma$. If $\gamma \in C_{M}$, then $M$ is $\gamma$-prime gamma ring if and only if $M$ is $\Gamma$-prime.

Proof. If $M$ is $\gamma$-prime gamma ring then $M$ is $\Gamma$-prime by Lemma 2.4. Let $M$ is a $\Gamma$-prime gamma ring, $a \gamma M \gamma b=0$ for any $a, b \in M$ and $a \neq 0$. Then we have $a \Gamma M \gamma M \gamma b=0$. Since $M$ is a $\Gamma$-prime $M \gamma M \gamma b=0$. Thus $M \gamma M \Gamma b \gamma M=0$. Hence we get $b=0$ since $M$ is a $\Gamma$-prime gamma ring and $\gamma \in C_{M}$. Therefore, $M$ is $\gamma$-prime.

4.4. Theorem. The prime radical of a $\Gamma$-ring $M$ is contained in $\gamma$-prime radical of $M$.

Proof. Let $x$ be an element of $\mathfrak{B}(M)$, the prime radical of $M$. Suppose that $x \notin \mathfrak{B}_{\gamma}(M)$. Then, there is a $\gamma$ - $m$-system $S$ which contains $x$ such that $0 \notin S$. Therefore, there is an $m$-system in $M$ which contains $x$ but not contains 0 since $S$ is also an $m$-system. This contradicts with $x \in \mathfrak{B}(M)$. Hence, if $x$ is an element of $\mathfrak{B}(M)$, then $x$ must be in $\mathfrak{B}_{\gamma}(M)$.

4.5. Theorem. The strongly nilpotent radical of a $\Gamma$-ring $M$ is contained in strongly $\gamma$-nilpotent radical of $M$.

Proof. It is easy to see that a strongly nilpotent ideal of $M$ is also a strongly $\gamma$-nilpotent ideal. Therefore, $\mathfrak{S}(M)$, the strongly nilpotent radical of $M$, is contained in $\mathfrak{S}_{\gamma}(M)$.

4.6. Theorem. The Levitzki nil radical of a $\Gamma$-ring $M$ is contained in $\gamma$-Levitzki nil radical of $M$.

Proof. It is easy to see that a locally nilpotent ideal of $M$ is also a $\gamma$-locally nilpotent ideal. Therefore, $\mathfrak{L}(M)$, the Levitzki nil radical of $M$, is contained in $\mathfrak{L}_{\gamma}(M)$.

4.7. Theorem. The Jacobson radical of a $\Gamma$-ring $M$ is contained in $\gamma$-Jacobson radical of $M$.

Proof. It is easy to see that a right quasi regular element of $M$ is also a $\gamma$-right quasi regular. Therefore, $\mathfrak{J}(M)$, the Jacobson radical of $M$, is contained in $\mathfrak{J}_{\gamma}(M)$.

4.8. Corollary. Let $M$ be a $\Gamma$-ring.

(i) If the ring $\left(M,+,{ }_{\gamma}\right)$ for any $\gamma \in \Gamma$ is semiprime, then the $\Gamma$-ring $M$ is $\Gamma$ semiprime.

(ii) If the ring $(M,+, \cdot \gamma)$ for any $\gamma \in \Gamma$ is semisimple, then the $\Gamma$-ring $M$ is $\Gamma$ semisimple.

\section{References}

[1] Arslan, O., Kandamar, H., $\gamma$-Lie structures in $\gamma$-prime gamma rings with derivations, J. Algebra Comb. Discrete Appl. 2 (1), 25-37, 2015.

[2] Awtar, R., Lie and Jordan structure in prime rings with derivations, Proc. Amer. Math. Soc. 41, 67-74, 1973

[3] Barnes, W. E., On the Г-rings of Nobusawa, Pacific J. Math. 18 (3), 411-422, 1966.

[4] Bergen, J., Kerr, J.W., Herstein, I.N., Lie ideals and derivations of prime rings, J. Algebra 71, 259-267, 1981.

[5] Coppage, W.E., Luh, J., Radicals of gamma rings, J. Math. Soc. Japan 23 (1), 40-52, 1971. 
[6] Herstein, I. N., A note on derivations, Canad. Math. Bull. 21 (3), 369-370, 1978.

[7] Herstein, I. N., A note on derivations II, Canad. Math. Bull. 22 (4), 509-511, 1979.

[8] Herstein, I. N., Topics in Ring Theory, The Univ. of Chicago Press, 132p., Chicago, 1969.

[9] Jing, F. J., On derivations of $\Gamma$-rings, Qu fu Shifan Daxue Xuebeo Ziran Kexue Ban 13 (4), 159-161, 1987.

[10] Kandamar, H., The k-Derivation of a Gamma-Ring, Turk. J. Math. 23 (3), 221-229, 2000.

[11] Kyuno, S., Gamma Rings, Hadronic Press, Palm Habor, 1991.

[12] Kyuno, S., On the radicals of gamma rings, Osaka J. Math. 12, 639-645, 1975.

[13] Lee, P. H., Lee T. K., On derivations of prime rings, Chinese J. Math. 9 (2), 107-110, 1981.

[14] Lee, P. H., Lee T. K., Lie ideals of prime rings with derivations, Bull. Inst. Math. Acad. Sinica 11 (1), 75-80, 1983.

[15] Luh, J., On the theory of simple $\Gamma$-rings, Michigan Math. J. 16 (1), 65-75, 1969.

[16] McCoy, N.H., The Theory of Rings, Chelsea Publishing Company, New York, 1973.

[17] Nobusawa, N., On a generalization of the ring theory, Osaka J. Math. 1, 81-89, 1964.

[18] Posner, E. C., Derivations in prime rings, Proc. Amer. Math. Soc. 8, 1093-1100, 1957.

[19] Rakhimov, I. S., Dey K. K., Paul A. C., On commutativity of completely prime gammarings, Malays. J. Math. Sci. 7 (2), 283-295, 2013. 